\title{
Edad y crecimiento del pez lobo Barbatula barbatula (Linnaeus, 1758) en un área de reciente introducción
}

\author{
M. Gutiérrez, J. Domínguez, y J.C. Pena \\ Departamento de Biología Animal. Facultad de Biología. Universidad de León. Campus de Vegazana 24071 León.
}

\section{RESUMEN}

Se estudia una población de pez lobo Barbatula barbatula (Linnaeus, 1758) introducida en un tramo de río Órbigo perteneciente a la cuenca del Duero, donde fue citado por primera vez en 1.988.

Se ofrecen los resultados del estudio de la edad y crecimiento de esta especie, realizado sobre 474 ejemplares, pertenecientes a una población cuyo seguimiento se ha realizado desde 1995 a 1999. La estimación de la edad ha sido realizada mediante la lectura de los otolitos, y el crecimiento mediante retrocálculo. Los ejemplares de mayor edad capturados pertenecen a la clase de edad 5 siendo todos ellos hembras. No existen diferencias significativas en la talla de los ejemplares de ambos sexos para las clases de edad representadas, La población estudiada está representada principalmente por individuos pertenecientes a las clases de edad $1+$ y $2+$.

Palabras clave: edad, crecimiento, pez lobo, otolito, población introducida, río Órbigo

\begin{abstract}
A population of stone loach, Barbatula barbatula (Linnaeus, 1758) was studied in a stretch of the River Órbigo íbasin of the Rivev Duero). At the site, this introduced species was first recorded in 1988.

We describe the age and growth of 474 loach specimens from a population studied between 1995 and 1999. Age determination was carried out using otoliths and the growth rate then back-calculated. The oldest group reached an age of 5 years and were allfemales. No statistically significant differences in length could be ohserved between males andfemales. The population was mainly composed by the $1+$ and $2+$ age groups
\end{abstract}

Keywords: age, growth, stone loach, otolith, Orbigo river.

\section{INTRODUCCIÓN}

La distribución natural del pez lobo, Barbatula barbatula (Linnaeus, 1758), en la Península Ibérica se limita a la cuenca del Ebro y al sector oriental de la vertiente cantábrica. Desde que en 1988 fuera localizado por primera vez en el río Órbigo, primera cita para la cuenca del Duero (González et al., 1989), es cada vez más frecuente en las aguas de este río, como se desprende del volumen de capturas que desde 1995, año en que se inicia el seguimiento de la población, se vienen efectuando.

Los posibles efectos que esta nueva especie introducida pueda ocasionar en la comunidad de peces preestablecida, ha motivado la realización de un estudio sobre su biología que, por otra parte, ha sido objeto de escasa atención en su área de distribución natural. Aquí se presentan los resultados relativos a la edad y crecimiento de esta especie.

\section{ÁREA DE ESTUDIO \\ El río Órbigo es fruto de la confluencia de dos ríos, el Luna y el Omaña, en el noroeste de la cuenca del Duero, a una altitud de 890 metros. Su cuenca constituye una de las principales redes hidrográfi-}


cas de la cuenca del Esla, drenando una superficie de $4972 \mathrm{~km}^{2}$ cuyo curso principal atraviesa la provincia de León de norte a sur hasta su desembocadura en el Esla, con un recorrido de $90 \mathrm{~km}$.

La cuenca del Órbigo, discurre sobre materiales silíceos con afloramientos de calizas en las zonas que corresponden al Luna (Alonso Herrero, 1995).

Los ejemplares objeto de este estudio se capturaron a la altura de la localidad de Huerga del Río (850 m) U.T.M. 30TTN669161, dentro del área ocupada por esta especie que se extiende por unos $80 \mathrm{~km}$ de cauce, entre las localidades de Villarrodrigo de Ordás, U.T.M. 30TTN688328 y Navianos de la Vega, U.T.M. 30TTM595884. (Fig. 1).

\section{MATERIAL Y MÉTODOS}

Los ejemplares fueron capturados entre abril de 1995 y julio de 1999, con periodicidad al menos estacional y mediante pesca eléctrica. Para ello

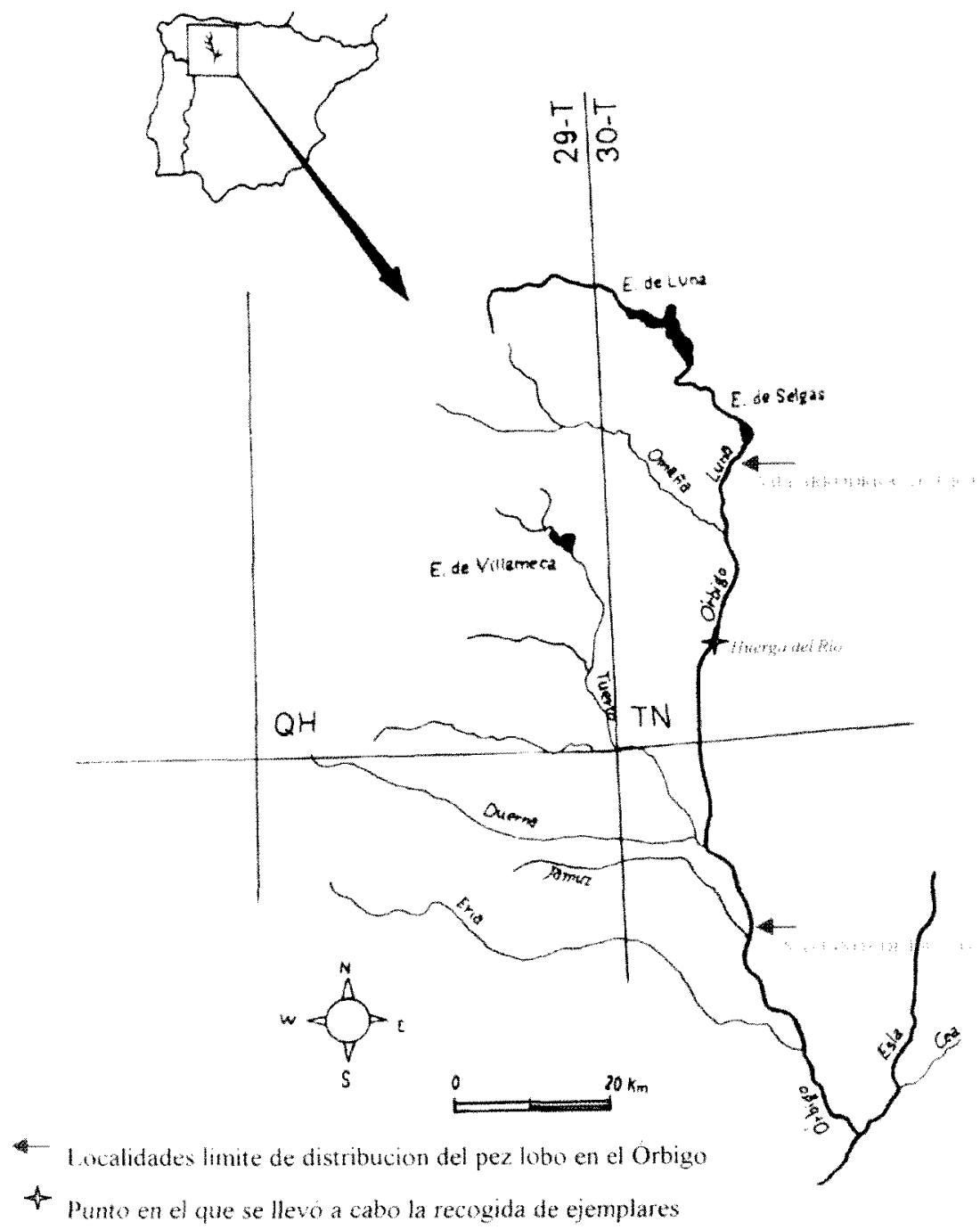

Figura 1. Distribución actual del pez lobo en el rio Órbigo. Actual distribution of the stone loach in the River Órbigo. 
fue utilizado un equipo de pesca eléctrica portátil "Martín Pescador" de la marca Erreka con electrodos móviles, con salida de corriente continua pulsada de voltaje variable y con intensidad entre 0,8 y $1,2 \mathrm{~A}$. El método utilizado fue el de esfuerzo de captura constante ejecutado por tres personas durante un tiempo de 15 minutos.

Se capturaron un total de 1335 ejemplares que fueron medidos, pesados y sexados. El sexo fue determinado por examen interno y para la determinación de la edad se recurrió a la lectura de los otolitos, dado que en esta especie las escamas son tan reducidas que no pueden ser utilizadas para su lectura. El par de otolitos más adecuado por su tamaño es el sagitta. De cada ejemplar se recolectaron los dos otolitos, derecho e izquierdo sobre los que fue realizada la lectura de la edad. Estos fueron extraídos de cada ejemplar mediante un corte transversal en la cabeza por detrás de los ojos. A continuación fueron limpiados suavemente con alcohol y almacenados en seco hasta el momento de su lectura.

El reducido tamaño de los otolitos permitió realizar la lectura de la edad sobre la pieza entera, por transparencia, sin necesidad de pulirlo o cortarlo. Para ello los otolitos se introducen entre 15 y 20 minutos, dependiendo del tamaño, en aceite de creosota lo que permite diferenciar las zonas de crecimiento al microscopio óptico (Smyly, 1955; Banks \& Irvine, 1969; Sauvonsaari, 1971; Liew, 1973; Williams \& Bedford, 1973, Robotham, 1981).

Las áreas alternativas opacas e hialinas observadas en forma de anillo bordeando el otolito se corresponden con las épocas de crecimiento rápido (primavera y verano) y lento (otoño e invierno), respectivamente.

Mediante la utilización de un microscopio óptico provisto de un micrómetro se tomaron medidas del diámetro total y de cada anillo de crecimiento estacional. Sobre una submuestra de 474 ejemplares seleccionados en función de la talla, se efectuó el retrocálculo de la longitud del pez a las distintas edades identificadas en el otolito (Robotham, 1981; Van Neer et al., 1999). Las longitudes medias para cada clase de edad y sexo así estimadas y las observadas directamente durante el periodo de mínimo crecimiento fueron comparadas mediante la utilización del test $t$.

Los parámetros de la ecuación de crecimiento de Von Bertalanffy fueron calculados mediante el método de Ford Walford y para determinar si existían diferencias de crecimiento entre machos y hembras fue aplicado el estadístico F propuesto por Ratkowsky para modelos no lineales (Chen et al., 1992)

La relación longitud-peso, se establece a través de la ecuación de la curva $\mathrm{W}=\mathrm{a} \mathrm{LT}^{\mathrm{b}}$ donde $\mathrm{W}$ es el peso en gramos. LT es la longitud total en milímetros, b es el coeficiente de alometría, y a es una constante (Smyly, 1955). La transformación logarítmica de esta expresión toma la forma $\log$ $\mathrm{W}=\log \mathrm{a}+\mathrm{b} \log \mathrm{L}$. Esta relación ha sido calculada, utilizando el total de los individuos capturados $(n=1335)$, separadamente para machos $y$ hembras. Para detectar las posibles diferencias en la relación longitud-peso entre sexos se realizó un análisis de covarianza y para determinar si el coeficiente de alometría, "b", se diferencia del valor teórico 3 que identifica el crecimiento isométrico en peso, se aplicó una $\chi^{2}$.

Por otra parte el índice de condición, índice ponderal o de Fulton, relaciona el peso y la longitud del pez y se utiliza para expresar el estado de éste, pudiendo observar diferencias ligadas al sexo o a la estación de captura. La expresión utilizada es: $\mathrm{K}=100 \mathrm{~W} / \mathrm{LT}^{3}$, siendo $\mathrm{W}$ el peso total en gramos y LT la longitud total en milímetros.

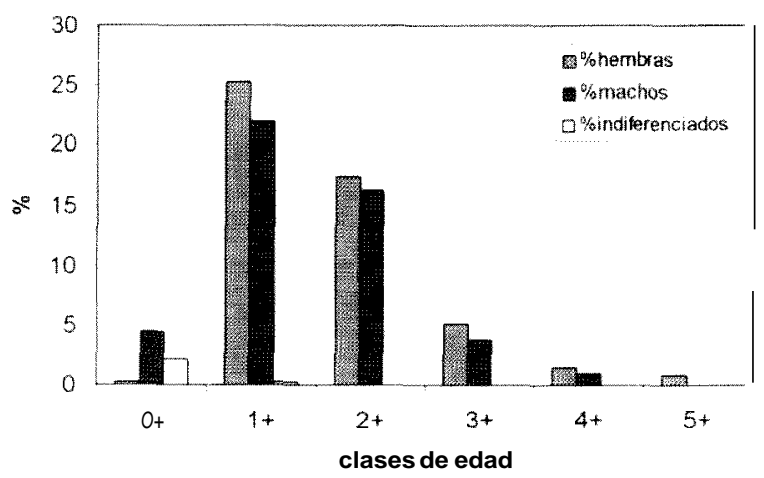

Figura 2. Porcentaje de hembras y machos distribuidos por clases de edad. Percentage of females and males in each age group. 


\section{RESULTADOS}

\section{Determinación de la edad y crecimiento}

De los 474 ejemplares sobre los que fue efectuada la lectura de la edad 256 eran hembras y 216 machos, alcanzando los ejemplares más longevos 5 años, todos ellos hembras.

Las clases de edad dominantes fueron las de $1+$ y $2+$. El número de ejemplares que corresponde a la clase de edad $0+$ es reducido, posiblemente influenciado, en parte, por el método de captura menos eficiente para individuos de talla inferior a de 30 milímetros (Lobón-Cerviá et al., 1991), estando esta clase de edad dominada por machos. (Fig. 2)

El proceso de determinación de la edad de los ejemplares ha permitido comprobar los periodos

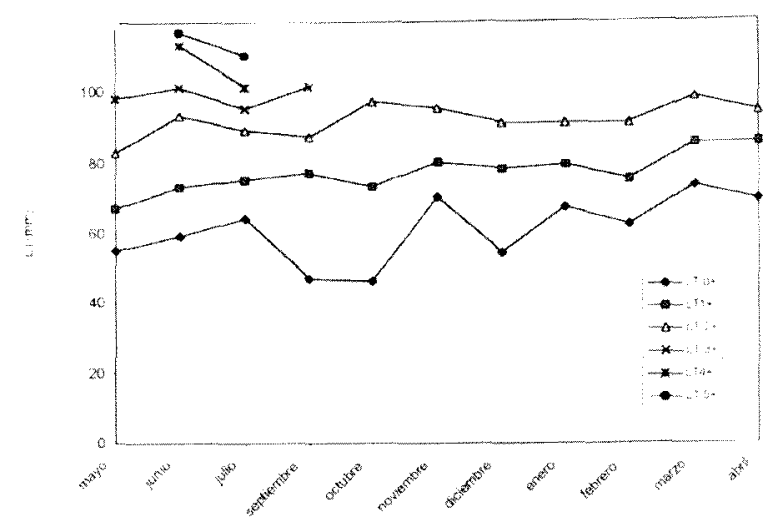

Figura 3. Crecimiento observado para cada clase de edad a lo largo del ciclo anual. Measured growth for different groups of age in the annual cycle de formación de los anillos de crecimiento en los otolitos. En el 69\% de los ejemplares capturados en el mes de mayo se ha detectado el inicio de formación de la porción opaca del anillo de crecimiento, correspondiente al periodo de máximo crecimiento. En el mes de diciembre se inicia la formación de la banda hialina en el borde exterior del otolito.

Puede asignarse el periodo de ralentización del crecimiento a los meses comprendidos entre diciembre y abril. El periodo de crecimiento rápido, por tanto, comprendería desde mayo a noviembre. $\mathrm{Si}$ a ello se añade que la población estudiada inicia la reproducción a finales del mes de abril (Gutierrez, 2002) se puede deducir que el pez lobo en este área completa el año de edad durante el mes de mayo, pudiendo tomarse como fecha de referencia el 15 de dicho mes.

El incremento en longitud (Fig. 3) se produce a partir del mes de mayo, si bien en el grupo de edad $0+$ se observa una disminución de la longitud media en los meses de septiembre y octubre, debido posiblemente a la incorporación de los ejemplares del año.

Entre el diámetro del otolito y la longitud total del pez, existe una correlación lineal y positiva altamente significativa $\mathrm{p}<0.001$, tanto para los machos como para las hembras. Las rectas de regresión estimadas por el método de los mínimos cuadrados toman la forma: LT hembras $=9.29+93.24$ diámetro $(\mathrm{r}=0.8)$ y LT machos $=$ $0.92+103.81$ diámetro $(\mathrm{r}=0.82)$, respectivamente.

Esto ha permitido estimar mediante retrocálculo las longitudes de los ejemplares estudiados en edades pretéritas. Los valores medios y de dis-

Tabla 1. Valores medios de la longitud total para los datos retrocalculados separados por sexos. El número de ejemplares es expresado entre paréntesis. DE es la desviación estándar. Se muestran los valores para el test de la t y la p obtenida. Mean total length for back-calculated and sexed data. The number of specimens is shown in brackets. "DE" are standard deviutions. t-test andp values are also shown.

\begin{tabular}{llllll}
\hline \multicolumn{5}{c}{ Media de la longitud total retrocalculada } \\
\hline EDAD & hembras (n) & DE & machos (n) & DE & Valor-t \\
1 & $61.88(77)$ & 9.6 & $63.76(66)$ & 8.49 & -1.21 \\
2 & $82.4(75)$ & 7.86 & $85.42(57)$ & 8.49 & -1.75 \\
3 & $96.53(39)$ & 6.08 & $98.12(24)$ & 7.39 & -0.92 \\
4 & $104.33(24)$ & 4.47 & $102.69(13)$ & 2.68 & 0.22 \\
5 & $107.3(3)$ & 5.57 & & & 0.35 \\
\hline
\end{tabular}


persión de las longitudes totales, para machos y hembras, en las sucesivas clases de edad obtenidas mediante retrocálculo, se muestran en la Tabla 1. La longitud media retrocalculada es ligeramente superior en los machos de 1, 2 y 3 años de edad que en las hembras coetáneas. Por el contrario cuando los individuos alcanzan su cuarto año, son las hembras las que presentan una longitud ligeramente mayor.

Los resultados del test demuestran que no existen diferencias significativas entre las medias de las longitudes totales $(p>0.05)$, para cada clase de edad y sexo

Las medias de las longitudes totales observadas durante el periodo de mínimo crecimiento, para ejemplares correspondientes a las distintas clases de edad y sexo, se muestran en la Tabla 2 junto con su desviación estándar. Al igual que para los datos obtenidos por retrocálculo se aplicó el t-test, no encontrándose diferencias significativas entre sexos $(p>0.05)$ en ninguna de las clases de edad estudiadas.

Dada la ausencia de diferencias entre las longitudes de los machos y de las hembras en cada clase de edad, se han podido tratar los datos en conjunto. Así, los valores medios de las longitudes totales retrocalculadas para cada clase de edad, fueron comparados con las longitudes totales medias reales observadas mediante t-test para cada clase de edad durante el periodo de menor crecimiento, no mostrando diferencias estadísticamente significativas $(\mathrm{p}>0.05)$ (Tabla 3$)$.
Se ha realizado la estimación de los parámetros de la ecuación de crecimiento utilizando los datos de las longitudes obtenidas mediante retrocálculo (Lobón-Cerviá et al. 1985; Velasco et al. 1990). Los parámetros obtenidos mediante el método de Ford Walford, separadamente para cada sexo han sido:

$\begin{array}{ll}\text { HEMBRAS } & \text { MACHOS } \\ \mathrm{L}_{\infty}=122 \mathrm{~mm} . & \mathrm{L}_{\infty}=109 \mathrm{~mm} . \\ \mathrm{K}=0.40 & \mathrm{~K}=0.67 \\ \mathrm{t}_{0}=0.105 & \mathrm{t}_{0}=0.072\end{array}$

No se encontraron diferencias significativas $(p>0.01)$ entre sexos para las ecuaciones de crecimiento calculadas al aplicar el test de la F. Los parámetros de la ecuación de crecimiento para el total de la población (Fig. 4) han sido:

$$
\begin{aligned}
& \mathrm{L}_{\infty}=121 \mathrm{~mm} . \\
& \mathrm{K}=0.45 \\
& \mathrm{t}_{0}=0.095
\end{aligned}
$$

\section{Relación longitud-peso}

Las ecuaciones de las rectas que relacionan la longitud y el peso para machos y hembras, estimadas por el método de los mínimos cuadrados son:

Log $\mathrm{W}$ hembras $=-5.35+3.15 \log \mathrm{LT}(\mathrm{r}=0.96)$ $\log \mathrm{W}$ machos $=-5.11+3.00 \log \mathrm{LT} \quad(\mathrm{r}=0.97)$

Tabla 2. Valores medios de la longitud total para los datos observados durante el periodo de menor crecimiento separados por sexos. El número de ejemplares es expresado entre paréntesis. DE es la desviación estandar. Se muestran los valores para el test de la t y la p obtenida. Mean total length calculated for the period of less growth. Data were grouped by sex. The number of individuals is shown in brackets. "DE" are standard deviations. t-test and p values are also shown.

\begin{tabular}{lllclc}
\hline \multicolumn{5}{c}{ Media de la longitud total retrocalculada } \\
\hline EDAD & hembras (n) & DE & machos (n) & DE & Valor-t \\
1 & $59.40(10)$ & 4.37 & $65.73(23)$ & 11.67 & -1.65 \\
2 & $82.40(60)$ & 7.91 & $85.46(47)$ & 7.91 & -1.97 \\
3 & $95.50(34)$ & 6.53 & $96.88(25)$ & 8.97 & -0.68 \\
4 & $103.39(23)$ & 3.95 & $102.68(16)$ & 2.46 & 0.63 \\
5 & $112.00(4)$ & 5.59 & & & 0.051 \\
\hline
\end{tabular}


Tabla 3. Medias de la longitud total obtenida de los ejemplares reales y mediante retrocálculo para el periodo de menor crecimiento. El número de ejemplares es expresado entre paréntesis. DE es la desviación estandar. Se muestran los valores para el test de la t y la p obtenida. Mean total length estimated from length of captured individuals and back-calculated. Lengths are for the period of less growth. The mumber of individuals is shown in bruckets. "DE" ure standard deviations. t-test and p values are also shown.

\begin{tabular}{lccccc}
\hline \multicolumn{2}{c}{ LT observada } & \multicolumn{2}{c}{ LT retrocalculada } & \\
EDAD & \multicolumn{1}{c}{ Media(n) } & DE & Media (n) & DE & Valor-t \\
\hline 1 & $61.68(38)$ & 11.16 & $62.67(146)$ & 9.06 & -0.56 \\
2 & $83.74(107)$ & 8.06 & $84.14(135)$ & 8.20 & -0.37 \\
3 & $96.08(59)$ & 7.62 & $97.35(65)$ & 6.64 & -0.99 \\
4 & $103.10(39)$ & 3.40 & $103.75(37)$ & 3.98 & -0.77 \\
5 & $112.00(4)$ & 5.59 & $107.3(3)$ & 5.57 & 0.70 \\
\hline
\end{tabular}

El análisis de covarianza aplicado, tomando la longitud como variable dependiente y el peso como covariable, muestra diferencias estadísticamente significativas (Tabla 4) entre sexos $(p<0.01)$.

Con el ánimo de evaluar las variaciones de la relación longitud-peso a lo largo del año, se realizó un nuevo análisis de covarianza entre sexos, tomando como variable dependiente el logaritmo

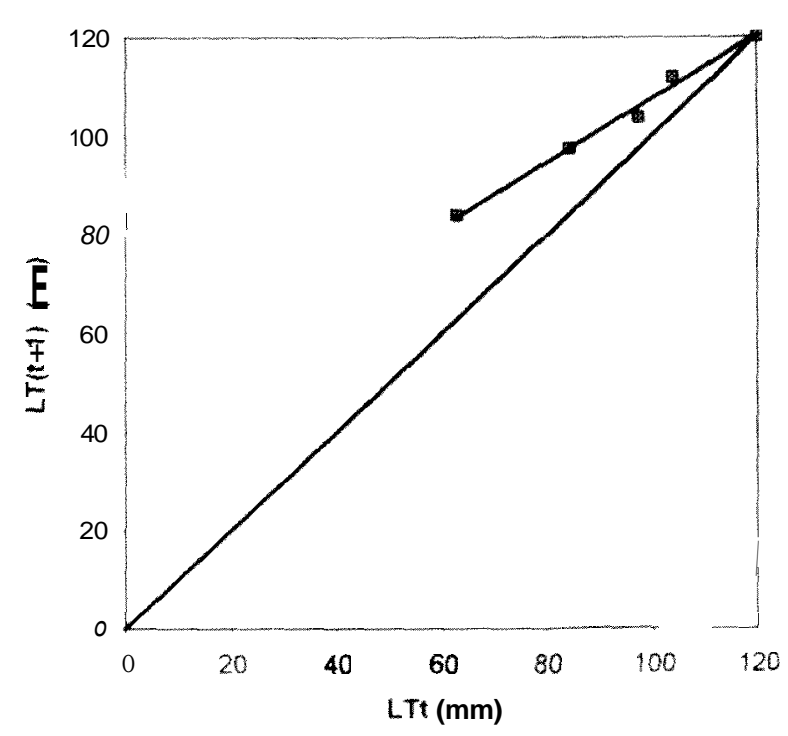

Figura 4. Fstimación de la ecuación de crecimiento mediante el método de Ford-Walford. Growth eauation estimated using the Ford-Walford method de la longitud total y el logaritmo del peso como covariable. Como puede advertirse, (Tabla 5), las diferencias entre sexos resultan ser Significativas desde los meses de febrero a julio. Estas diferencias pueden ser atribuidas a la influencia del desarrollo gonadal diferenciado en dicho periodo.

Los valores de los coeficientes de alometría para los dos sexos, se muestran en la Tabla 6. La variación de b a lo largo del año se presenta en la figura 5. La prueba $\chi^{2}$ realizada no mostró diferencias significativas con el valor teórico 3 que representa el crecimiento isométrico.

El periodo del año en el que los valores del coeficiente de alometría se mantienen más bajos, es coincidente con la época en la que se encuentra una disminución en el número de estómagos con contenido y de reposo gonadal. (Cutiérrez, 2002).

\section{Índice de condición}

Este índice ha sido calculado para machos y hembras por separado. Los valores del índice de condición $\mathrm{K}$, a lo largo del año, alcanzan un máximo en mayo y decaen durante el verano, coincidiendo con el periodo reproductor, recuperándose de nuevo a partir de noviembre.

En el caso de las hembras la variación es más pronunciada debido a la influencia del peso de la gónada femenina. En el caso de los machos las fluctuaciones no son tan marcadas. (Fig. 6) 
Tabla 4. Resultados del ANCOVA del peso en función de la longitud y el sexo de los ejemplares. Results of the ANCOVA separating weigth. total length and sex

\begin{tabular}{lcccc}
\hline & Grados de libertad & Suma de cuadrados & F & P \\
\hline Sexo & 1 & 0.047 & 205.51 & 0.00 \\
Error & 1282 & 0.0002 & & \\
\hline
\end{tabular}

\section{DISCUSIÓN}

No existen muchas publicaciones relativas a edad y crecimiento del pez lobo. En lo que se refiere a la longevidad es este un aspecto sobre el que existe más información de referencia. Se ha intentado relacionar la longevidad con el tipo de masa de agua de procedencia de las poblaciones: lagos, arroyos y ríos, sin embargo los resultados no son concluyentes puesto que mientras los trabajos de Smyly (1955) sugerían que el pez lobo en ríos y arroyos alcanzaba los 5 años de edad y no superaba los 3 años en lagos, los resultados de Milla \&
Welton (1983), Sauvonsaari (1971) y Mills \& Eloranta (1985) parecen demostrar lo contrario. Concretamente el primero de estos estudios registra una edad máxima de 3 años en el río Frome, área próxima a aquella estudiada por Smyly (1955). Por su parte los trabajos referidos de Sauvonsaari y Mills \& Elonrata apuntan la edad de 6 años en lagos de Finlandia. En nuestro caso, para la población del río Órbigo estudiada la clase de edad más elevada se sitúa en 5 años, perteneciente al $0.84 \%$ del total de los ejemplares estudiados.

Otra explicación a estas diferencias se ha buscado por estos y otros autores en la conductividad

Tabla 5. Resultados del ANCOVA realizado por meses entre sexos. Significación de las diferencias:** $(\mathrm{p}<0,01),{ }^{* *}(\mathrm{p}<0,001)$. Results of the ANCOVA separated by month and sex. Significance of differences $* *(p<0.01),{ }^{* * *}(p<0.001)$.

\begin{tabular}{|c|c|c|c|c|c|}
\hline Mes & & $\begin{array}{l}\text { Grados } \\
\text { de libertad }\end{array}$ & $\begin{array}{l}\text { Suma } \\
\text { de cuadrados }\end{array}$ & F & $\mathrm{P}$ \\
\hline \multirow[t]{2}{*}{ Enero } & Sexo & 1 & 0.00008 & 0.34 & 0.5638 \\
\hline & Error & 32 & 0.00024 & & \\
\hline \multirow[t]{2}{*}{ Febrero } & Sexo & 1 & 0.00254 & 16.6 & $0.0004^{* * *}$ \\
\hline & Error & 26 & 0.00015 & & \\
\hline \multirow[t]{2}{*}{ Marzo } & Sexo & 1 & 0.01203 & 74.63 & $0.0000 * * *$ \\
\hline & Error & 149 & 0.00016 & & \\
\hline \multirow[t]{2}{*}{ Abril } & Sexo & 1 & 0.03260 & 148.56 & $0.0000 * * *$ \\
\hline & Error & 193 & 0.00021 & & \\
\hline \multirow[t]{2}{*}{ Mayo } & Sexo & 1 & 0.00884 & 52.11 & $0.0000^{* * *}$ \\
\hline & Error & 42 & 0.00017 & & \\
\hline \multirow[t]{2}{*}{ Junio } & Sexo & 1 & 0.01649 & 29.16 & $0.0000^{* * *}$ \\
\hline & Error & 329 & 0.00056 & & \\
\hline \multirow[t]{2}{*}{ Julio } & Sexo & 1 & 0.00149 & 10.22 & $0.0016^{* *}$ \\
\hline & Error & 167 & 0.00014 & & \\
\hline \multirow[t]{2}{*}{ Septiembre } & Sexo & 1 & 0.00007 & 0.55 & 0.4573 \\
\hline & Error & 192 & 0.00012 & & \\
\hline \multirow[t]{2}{*}{ Octubre } & Sexo & 1 & 0.00012 & 1.64 & 0.2092 \\
\hline & Error & 32 & 0.00007 & & \\
\hline \multirow[t]{2}{*}{ Noviembre } & Sexo & 1 & 0.000001 & & \\
\hline & Error & 42 & 0.00016 & 0.0083 & 0.9278 \\
\hline \multirow[t]{2}{*}{ Diciembre } & Sexo & 1 & 0.00006 & 8.22 & 0.0061 \\
\hline & Error & 48 & 0.00053 & & \\
\hline
\end{tabular}




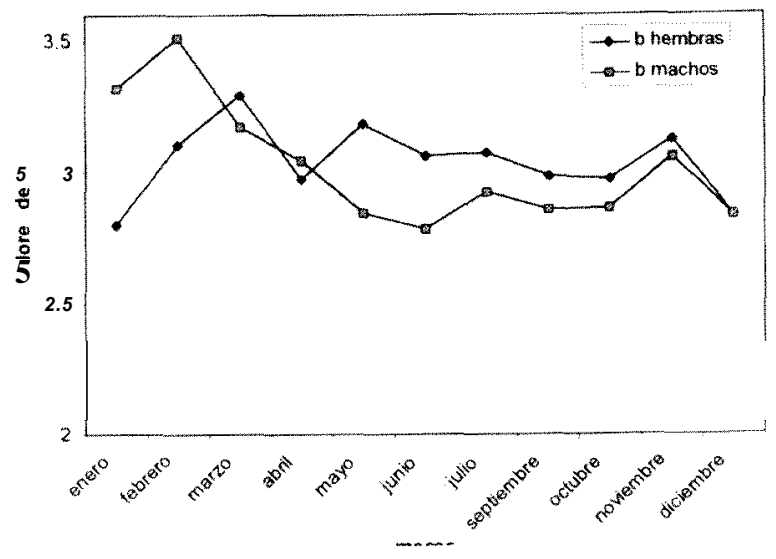

Figura 5. Variación de b para machos y hembras a lo largo del año Annual changes in $b$ of males and females

del agua. Mills \& Welton (1983) asocian los ejemplares más longevos con aguas de escasa conductividad. Es decir los peces lobo de 5 años de edad estudiados por Smyly (1955) pertenecen a arroyos de baja conductividad en tanto que los del río Frome, de aguas más salinas sólo alcanzan los 3 años de vida. Esto serviría para explicar por qué nuestros ejemplares alcanzan unos valores más elevados, ya que la conductividad del agua en la zona de estudio se situó entre los 112 y 214 $\mathrm{ms} / \mathrm{cm}$, menores que las registradas por Zweimüeller (1995) y Mastrorillo et al. (1996).

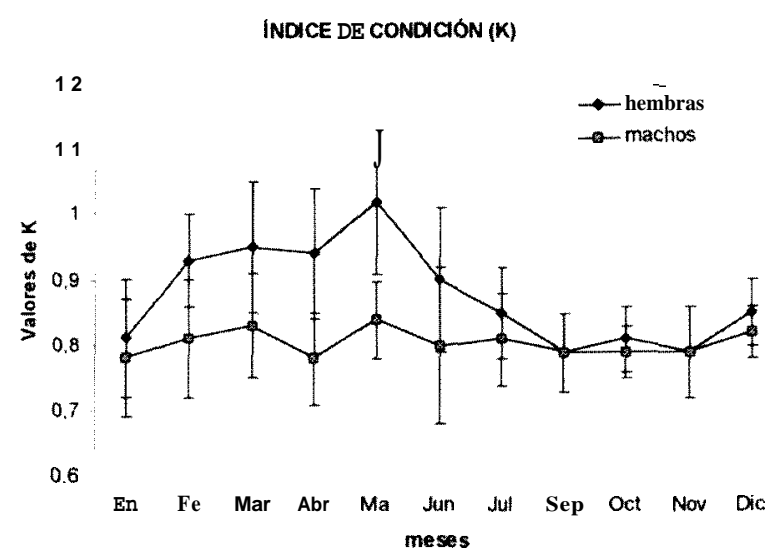

Figura 6. Ciclo anual del índice de condición para machos y hembras. Annual cycle of the condition index in males and females
No obstante, y debido al corto periodo de aclimatación del pez lobo en el área de estudio no debe descartarse la aparición de ejemplares más longevos en el transcurso del tiempo de permanencia en el río Órbigo.

El periodo de máximo crecimiento, establecido entre mayo y noviembre, resultó más extenso que el expuesto por Mills et al., (1983) en el río Frome (Inglaterra), entre mayo y octubre, cuando la temperatura del agua excede $\operatorname{los} 12^{\circ} \mathrm{C}$, hecho lógico ya que en el área del presente trabajo, más meridional, dicha temperatura es superada durante un periodo más dilatado de tiempo a lo largo del año.

Por otra parte el hecho de que el máximo crecimiento se inicie en primavera, además del incremento de temperatura, puede verse influenciado porque es el momento en el que la tasa de ingesta, medida como proporción de estómagos con contenido, es la más elevada durante el año (Gutiérrez, 2002).

En cuanto a las longitudes medias de cada clase de edad, si bien no existen diferencias estadísticamente significativas entre los sexos, se aprecia una ligera desviación hacia los machos en los primeros años de vida. Esto mismo señalan Smyly (1955), y Mills \& Welton (1983). Estos últimos añaden que puede estar influenciado por la dificultad para sexar los ejemplares jóvenes. En lineas generales la talla alcanzada por los ejemplares más jóvenes en el presente estudio se aproxima a aquellas referidas para ríos y arroyos y los más longevos se aproximan a las poblaciones lacustres de referencia.

Por lo que se refiere al incremento en longitud interanual lógicamente el máximo se corresponde con los primeros años de vida, coincidiendo con los resultados de Smyly (1955), Sauvonsaari (1971) y Penczak et al. (1981).

Si nos referimos a la relación longitud-peso, las variaciones que se producen a lo largo del año en el coeficiente de regresión son debidas, según Bagenal (1971), al sexo, grado de madurez o incluso al llenado de los estómagos. Los valores más altos alcanzados por el coeficiente de alometría se sitúan, al igual que los correspondientes al mayor porcentaje de individuos con contenido estomacal, en primavera. 
Tabla 6. Coeficientes de las rectas de regresión longitud-peso $\log W=\log \mathrm{a}+\mathrm{b} \log \mathrm{LT}$, a lo largo del año para machos y hembras. Least-squares regressioncoefficients for the length-weight relationships $\log W=\log u+b \log L$ T estimated for all-year data, for males and females.

\begin{tabular}{|c|c|c|c|c|c|c|c|c|}
\hline \multirow[b]{2}{*}{ Meses } & \multicolumn{3}{|c|}{ Hembras } & \multirow[b]{2}{*}{$\mathrm{r}$} & \multirow[b]{2}{*}{$\mathrm{n}$} & \multicolumn{3}{|c|}{ Machos } \\
\hline & $\mathrm{n}$ & $\log a$ & $\mathrm{~b}$ & & & $\log a$ & $\mathrm{~b}$ & $\mathrm{r}$ \\
\hline Enero & 23 & -4.72 & 2.80 & 0.95 & 12 & -5.72 & 3.32 & 0.95 \\
\hline Febrero & 17 & -5.23 & 3.10 & 0.98 & 12 & -6.07 & 3.51 & 0.98 \\
\hline Marzo & 83 & -5.59 & 3.29 & 0.96 & 69 & -5.41 & 3.17 & 0.98 \\
\hline Abril & 106 & -4.96 & 2.97 & 0.96 & 90 & -5.19 & 3.04 & 0.97 \\
\hline Mayo & 23 & -5.34 & 3.18 & 0.98 & 22 & -4.78 & 2.84 & 0.99 \\
\hline Junio & 201 & -5.17 & 3.06 & 0.95 & 131 & -4.69 & 2.78 & 0.88 \\
\hline Julio & 90 & -5.22 & 3.07 & 0.97 & 80 & -4.95 & 2.92 & 0.97 \\
\hline Septiembre & 93 & -5.06 & 2.98 & 0.96 & 102 & -4.82 & 2.85 & 0.96 \\
\hline Octubre & 24 & -5.03 & 2.97 & 0.98 & 11 & -4.84 & 2.86 & 0.97 \\
\hline Noviembre & 27 & -5.34 & 3.12 & 0.94 & 18 & -5.20 & 3.05 & 0.93 \\
\hline Diciembre & 25 & -4.75 & 2.83 & 0.99 & 26 & -4.78 & 2.83 & 0.99 \\
\hline
\end{tabular}

Las fluctuaciones observadas en el índice de condición $\mathrm{K}$ a lo largo del año, se ven influenciadas, fundamentalmente, por el peso de las gónadas, de esta manera se presentan en las hembras mucho más marcadas que en los machos. En los estudios referidos por Mills et al., (1983) y Mills \& Eloranta (1985), el máximo para los machos se presenta en marzo, apareciendo los valores más bajos al final del verano. También en el presente estudio se ha observado que los valores máximos para los machos se presentan entre marzo y mayo. El índice, en el caso de las hembras, aumenta rápidamente al principio de la primavera, manteniéndose constante en marzo y abril alcanzando el máximo en junio, descendiendo después rápidamente una vez iniciada la freza. El mismo caso ha sido reseñado para otras especies, como el gobio (Lobón-Cerviá et al., 1991; Prokes et al., 1988). En el primer caso el estudio se realizó en un área de reciente colonización, mientras que el segundo correspondía al área de distribución natural. Hyslop (1982) relaciona la disminución en el índice de condición durante los meses de otoño, con una disminución en la alimentación, de igual forma se ha observado a lo largo del presente estudio una tasa de ingesta más reducida durante los meses de otoño.

\section{BIBLIOGRAFÍA}

ALONSO HERRERO, E. 1995.Atlas del medio natural de la provincia de León. I.T.G.E. Diputación de León. Madrid 103 pp.

BAGENAL, T. B. 1971. The interrelation of the size of fish egg, the date of spawning and the production cycle. J. Fish Biol., 3: 207-2 19.

BANKS, J. W. \& W. IRVINE. 1969A note on the photography of fish scales, opercular and otholiths using an enlarger.J. Fish Biol., $1: 25-26$.

CHEN, Y., D. A. JACKSON, \& H. H. HARVEY. 1992. A comparison for Von Bertelanffy and polynomial functions in modelling fish growth data. Can. J Fish. and Aquat. Sci., 49: 1228-1235.

DOADRIO, I. (Ed.) 2001. Atlas y Libro Rojo de los Peces Continentales de España. Dirección General de Conservación de la Naturaleza. Museo Nacional de Ciencias Naturales. Madrid 364 pp.

GARCÍA DE JALÓN, D., M. MAYO, F. HERVELLA, E. BARCELÓ, \& T. FERNÁNDEZ. 1993. Principios y técnicas de gestión de la pesca en aguas continentales. Ed: Mundi Prensa. Madrid. 247 pp.

GONZÁLEZ, G., J. DOMINGUEZ, \& J. C. PENA. 1989. Primera cita de Noemacheilus barbatulus L. 1758 en la cuenca de Duero. Misc. Zool., 13:201-202. GUTIERREZ, M. 2002. Estudio de la biología del pez lobo Barbatula barbatula (Linnaeus, 1758) en unárea de reciente colonización (Rio Órbigo, León). Tesis Doctoral Universidad de León. 259 pp. 
HYSLOP, E. J. 1982. The feeding habits of 0+ ctone loach, Noemacheilus barbatulus (L.) and bullhead, Cottus gobio L. J. Fish Biol.,21: 187-196.

LIEW, P. K. L. 1973. Age determination of American eels based on the structure of their otoliths. En: The ageing of fish. T.B. Bagenal (ed.). Surrey. 234pp.

LOBÓN-CERVIÁ, J., P. ROVIRA, \& O. SORIANO. 1985. Contribución a la biología y biometría de la bermejuela Rutilus arcassi (Steindch., 1866) del embalse de Pinilla. Doñana Acta Vertebrata., 12(1): 5-19.

LOBÓN-CERVIÁ, J. C. MONTAÑES \& A. DE SOSTOA. 1991. Influence of environment upon the life history of gudgeon, Gobio gobio (L.): a recent and successful colonizer of the lberian Peninsula. $J$ Fish Biol.. 39: 285-300.

MASTRORILLO, S. F. DUBA \& A. BELAUD. 1996. Utilisacion des microhabitat par le vairon le goujon et loche franche daus trois riviers du sud-ouest de la France. Annls. Limnol., 32 (3): 185-195.

MILLS, C. A. \& A. ELORANTA. 1985. Reproductive strategies in the stone loach, Noemacheilus barbatulus. Oikos, 44: 341-349.

MILLS, C. A. J. S. WELTON \& E. L. RENDLE. 1983. The age growth and reproduction of the stone loach, Noemacheilus barbatulus (L.) in a Dorset chalk stream. Freshwat. Biol., 13: 283-292.

PENCZAK, T., M. ZALEWSKI, E. SUSZYCKA \& M. MOLINSKI. 1981. Estimation of the density biomass and growth rate of fish population in two small lowland rivers. Ekologia Polska, 29 (2): 233-255.
PEREIRO, J. A. 1982. Modelos al uso en dinúmica de poblaciones marinas sometidas a explotación. instituto Español de Oceanografía. 1:254pp.

RINCÓN, P. A. \& J. LOBÓN-CERVIÁ 1989. Reproductive and growth strategies of the red roach, Rutilus arcassi (Steindachner, 1866), in two contrasting tributaries of the river Duero, Spain. $J$ Fish Biol., 34: 687-705.

ROBOTHAM, P. W. J. 1981. Age growth and reproduction of a population of spined loach Cobitis taenia (L.). Hydrobiologia., 85: 129-136.

SAUVONSAARI, J. 1971. Biology of the stone loach (Nemacheilus barbatulus L.) in the lakes Paijanne and Palkanevei Southern Finland. Ann. Zool. Fennici, 8: 187-193.

SMYLY, W. J. P. 1955. On the biology of the stone loach Nemacheilus barbatula L. The Journal of Animal Ecology, 24 (1): 167-186.

VAN NEER, W., L. LOUGAS \& A. D. RINSDORP. 1999. Reconstructing age distribution, season of capture and growth rate of fish from archaelogical sites based on otoliths and vertebrae. Int. J. Osteoarchael., 9: 116-130.

WEATHERLEY, A. H. 1972. Growth and ecology of fish populations. Academic Press. London. 293 pp.

WILLIAMS, T.\& B. C. BEDFORD. 1973. The use of otholiths for age determination. In: The ageing of fish. T. B. Bagenal (ed.) Surrey. 234 pp.

ZWEIMÜELLER, I. 1995. Microhabitat use by two small benthic stream fish in a second order stream. Hydrobiologia, 303: 125-137. 